\title{
V2V-Intersection Management at Roundabouts
}

\author{
Reza Azimi, Gaurav Bhatia and Ragunathan (Raj) Rajkumar
}

Carnegie Mellon University

Priyantha Mudalige

General Motors

\begin{abstract}
More than $44 \%$ of all automotive crashes occur in intersections. These incidents in intersections result in more than 8,500 fatalities and approximately 1 million injuries each year in USA [1,18]. It is also established that roundabouts are safer than junctions. According to a study of a sampling of roundabouts in the United States, when compared with the junctions they replaced, roundabouts have $40 \%$ fewer vehicle collisions, $80 \%$ fewer injuries and $90 \%$ fewer serious injuries and fatalities [14].

In earlier work [2,12], we have proposed a family of vehicular network protocols, which use Dedicated Short Range Communications (DSRC) and Wireless Access in Vehicular Environment (WAVE) technologies to coordinate a vehicle's movement through intersections. We have shown that vehicle-to-vehicle (V2V) communications can be used to avoid collisions at the intersection and also significantly decrease the trip delays introduced by traffic lights and stop signs. In this paper, we investigate the use of our proposed $\mathrm{V} 2 \mathrm{~V}$-intersection protocols for autonomous driving at roundabouts. We have extended our hybrid emulator-simulator called AutoSim to implement realistic map and mobility models to study traffic flow at roundabouts and have implemented our V2Vintersection protocols on roundabouts. We quantify the improvement in safety and throughput when our intersection protocols are used to traverse roundabouts.
\end{abstract}

\section{INTRODUCTION}

Based on the statistics collected from the Federal Highway Administration (FHWA) and National Highway Traffic Safety Administration (NHTSF), approximately 3 million vehicle crashes occur at intersections which are currently managed by stop signs and traffic lights $[1,18]$. The future of transportation points towards autonomous driving as a way of reducing fatalities and optimizing traffic flow management. Within this context, we advocate the use of vehicular networks to design distributed protocols in which vehicles are able to interact with each other using vehicle-to-vehicle (V2V) communications. Our goal is to enhance safety while decreasing the delay introduced by stop signs or traffic lights by using our $\mathrm{V} 2 \mathrm{~V}$-based intersection management protocols.

Some related work with the goal of managing the traffic at intersections using Vehicle-to-Infrastructure (V2I) communications has been done in the past few years $[3,4,5,6,7,19,20,21]$. The cost of installing the infrastructure required for the V2I approach on each and every intersection is significantly high, and makes it somewhat impractical. Another drawback is that this approach is based on a centralized system in which the infrastructure is the single point of failure and in the case that it fails, vehicles must somehow coordinate on their own. To address these shortcomings, we proposed that Vehicle-to-Vehicle (V2V) communications can be used as a part of a distributed system in which all the approaching vehicles are interacting with each other and make decisions about when to cross the intersection safely and efficiently. Our focus in this paper is to (a) improve our V2V-based intersection management protocol and introduce a new Collision Detection Algorithm for Roundabouts (CDAR), (b) extend our hybrid emulator-simulator AutoSim, by introducing Route Network Definition File (RNDF) files and constructing roundabout routes based on the GPS coordinates extracted from digital map databases, and (c) compare the operational efficiency of our protocols on roundabouts with our previous work and conventional traffic lights.

The rest of this paper is organized as follows. Section 2 presents the Collision Detection Algorithm for Roundabouts $($ CDAR) used in our new intersection protocol. Section 3 describes the characteristics of roundabouts. Section 4 contains a brief summary of our V2Vbased protocols: Concurrent Crossing-Intersection Protocol (CC-IP) and Maximum Progression-Intersection Protocol (MP-IP) and the modifications to make them suitable for managing the traffic through roundabouts. In Section 5, we present the evaluation of our protocols using AutoSim. Section 6 presents our concluding remarks and future work. 


\section{ROUNDABOUTS}

A roundabout is a channelized intersection with one-way traffic flow circulating around a central island. Roundabouts are also considered as "traffic-calming" devices since all traffic is slowed to the design speed of the one-way circulating roadway. The average velocity allowed at roundabouts is between 15 and $25 \mathrm{mph}$. These slower speeds reduce the severity of crashes, and minimize the total number of all crashes inside the roundabout area. Roundabouts are small, generally from 70 to 160 feet in diameter compared to 300 to 400 feet and more for traffic circles and rotaries. Roundabouts have a distinct feature of raised entry splitter islands which constrain vehicle speeds just before entry [13,14].

The Federal Highway Administration reports that, in just one recent year, approximately one death occurred every hour nationwide relating to intersections. Over nine thousand people lost their lives in traffic intersections in that recent year, equaling nearly one quarter of all traffic fatalities and amounting to a financial loss of over $\$ 96$ billion [22]. In addition to slower speeds, roundabouts have fewer conflict points than traditional intersections. Figure 1 shows a comparison of conflict points between a single-lane roundabout and a single-lane perfect cross intersection. Note that the cross intersection includes 32 conflict points while the number of conflict points at the roundabout is only a quarter of that number, meaning 8 points.
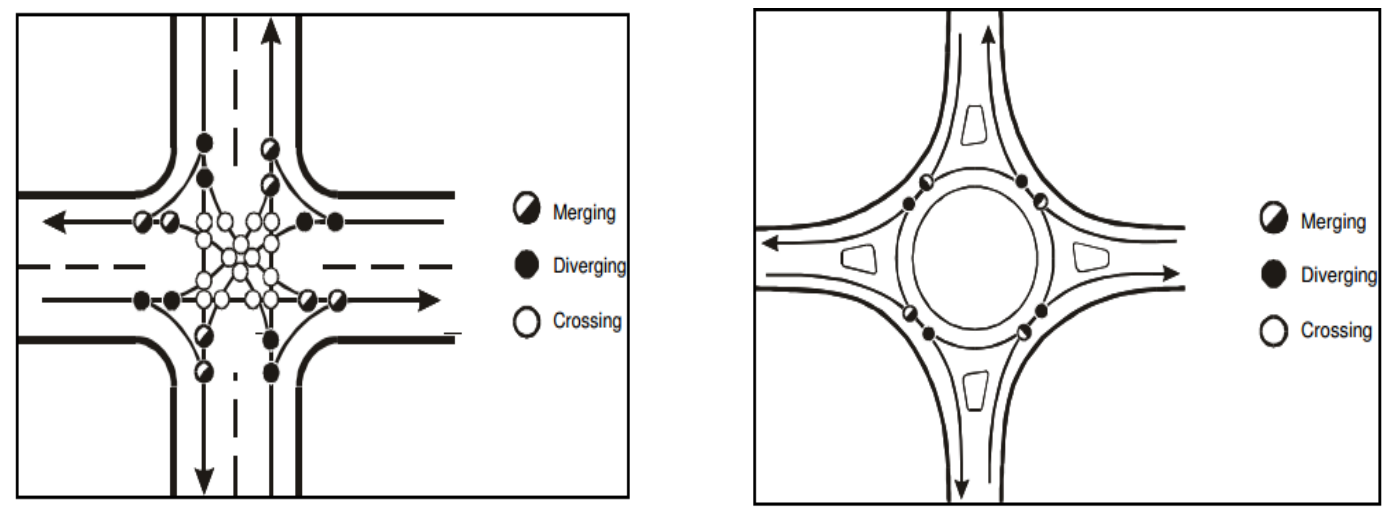

Figure 1, Traffic conflicting points at a simple cross intersection and a 1-lane roundabout [16]

Figure 2 shows that, in multiple-lanes roundabouts, the number of conflict points increases, but it is still much less than the number of conflict points compared to a 2-lane cross intersection.
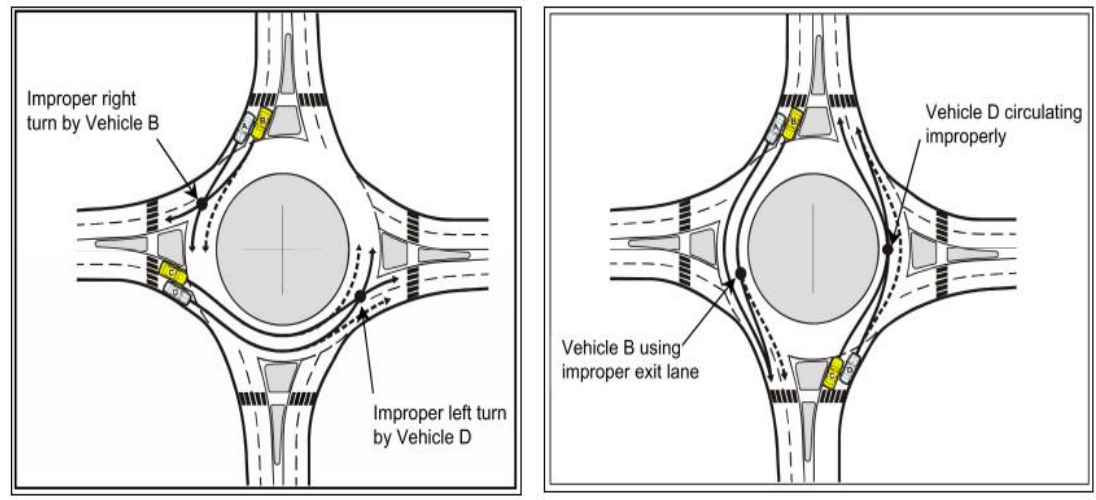

Figure 2, Additional conflicting points at multi-lane roundabouts

In addition to the safety benefits of roundabouts, the slower circulating speeds at roundabouts allow entering vehicles to accept smaller gaps in the circulating traffic flow, meaning more gaps are available, increasing the volume of traffic processed. Coming to a complete stop only happens when there is not enough gap due to the high density of traffic. However, when dealing with low traffic volumes, due to the continuously flowing nature of yielding only until a gap is available, roundabouts outperform traffic lights in regard to total throughput and capacity. 


\section{COLLISION DETECTION AT INTERSECTIONS}

We currently define the roundabout as a channelized intersection, in which four roads converge towards a central island from different directions. Each road includes predefined entry and exit points for each lane connected to it. The roundabout area is considered to be a grid which is divided into small cells. Each cell in the roundabout grid is associated with a unique identifier. Figure 3 shows a 1-lane roundabout. Note that the roundabout grid is divided into eight small cells and each of them is big enough to fit an average sized vehicle $(4.5 m * 2.5 m)$ in it. The cell geometry and number of cells is unique for each particular roundabout and depends on physical parameters of the roundabout area, such as the radius of the intersection and the size of its central island. Cell geometry is also determined based on the number of lanes entering the roundabout from each direction.

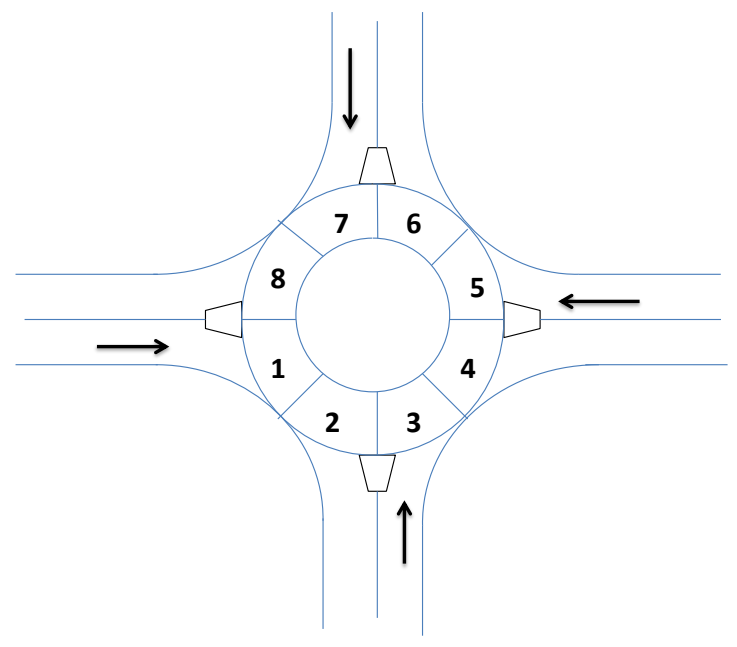

Figure 3, Roundabout grid divided into small cells

We make the following assumptions. Each vehicle has access to a digital map database that provides road and lane information. Intersections are identified by unique identifiers (IDs) on this map. Intersections have well-defined approach and exit lanes. Vehicles also have access to a global positioning system (GPS) with locally generated Radio Technical Commission for Maritime (RTCM-104) corrections to obtain a Real-time Kinematic (RTK) solution in order to achieve reliable lane-level vehicle positioning. Such GPS augmentation can be made available by local base stations or through commercial service providers.

We define the current road segment (CRS) as the road segment that a vehicle is on before the roundabout, and the next road segment (NRS) represents the road segment that the vehicle will be on after crossing the roundabout.

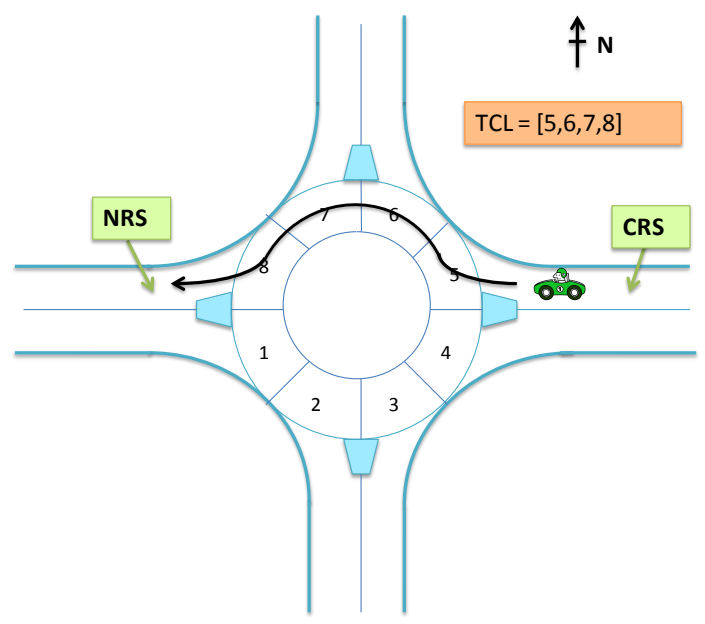

Figure 4, Illustration of TCL, CRS and NRS

Page 3 of 13 
Each vehicle uses the information about the CRS, NRS and the roundabout's cell geometry to create a list including the cells that will be occupied by the vehicle while crossing the roundabout. This list is called the Trajectory Cells List (TCL). The TCL is sorted based on the order of cells that are entered by the vehicle. Figure 4 shows the scenario in which the vehicle is entering the roundabout from the east and going to the west. Based on the CRS, NRS and the cell geometry, the vehicle builds its TCL as $\{5,6,7,8\}$. As mentioned, all the vehicles are supposed to be equipped with GPS devices and have access to the digital map database as well as the roundabout's coordinates. Therefore, each vehicle is able to use this information to map the current GPS coordinates to its current cell number. The current cell number will be then used to update the TCL and will be broadcast to surrounding vehicles as part of the basic safety message (BSM) [11]. Each vehicle uses the information about its current cell to update the TCL while crossing. Specifically when the vehicle leaves one cell and enters a new cell, the previous current cell gets deleted from the TCL. The updated TCL is useful for neighboring vehicles as it gives them explicit information about the cells which are not occupied anymore and might be used by other vehicles to cross the roundabout.

Our Collision Detection Algorithm for Roundabouts (CDAR) runs on all vehicles, using the information obtained from received safety messages, which are broadcast by surrounding vehicles. The algorithm uses the Trajectory Cells Lists of the sender and the receiver of the safety messages and by comparing the two lists, it determines if there is any common cell along their trajectories while crossing the roundabout. If a potential collision is detected by CDAR, the algorithm returns the first conflicting cell number which we refer to as the Trajectory-Intersecting Cell (TIC).

Figure 5 shows two example scenarios, in which two vehicles are crossing the roundabout at the same time. In Figure 5(a), one of the vehicles has the TCL of $\{1,2,3,4\}$ and the other one's TCL is $\{5,6,7,8\}$. As there is no common cell along their trajectories, they are able to enter and cross the roundabout at the same time without the potential for any collision. On the other hand, Figure 6 illustrates another roundabout-crossing scenario, where there are common cells along crossing vehicles' TCLs and potential collisions may occur. In the example scenario of 6(a), the CDAI running on both vehicles will inform them that cell number 5 is the first Trajectory Intersecting Cell (TIC) along their trajectories. And in the case of Figure 6(b), cell number 7 is the first TIC of the crossing vehicles.

(a)

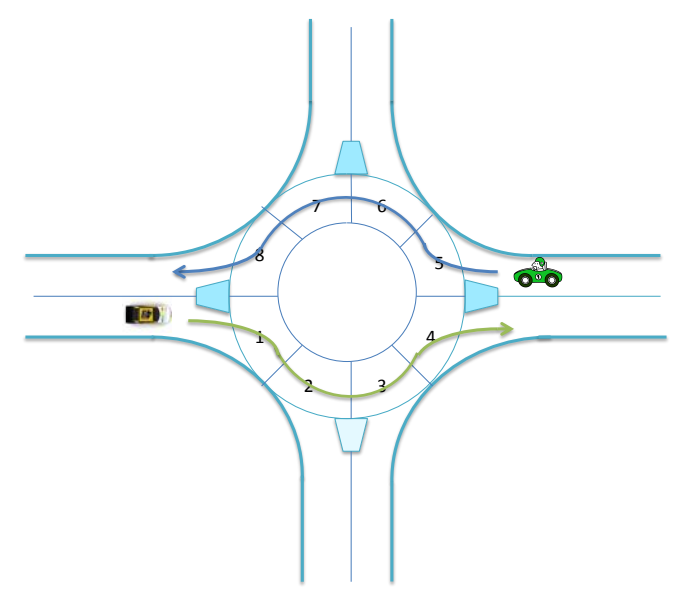

(b)

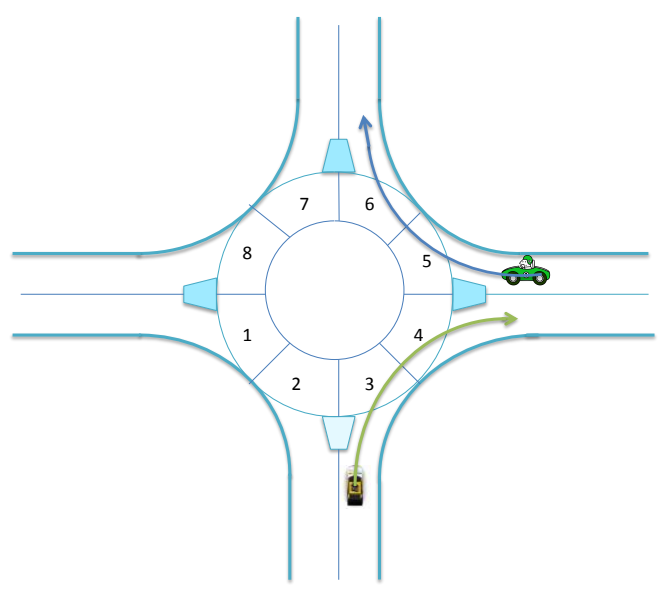

Figure 5, Example scenario in which no space conflict occurs at the roundabout

(a)

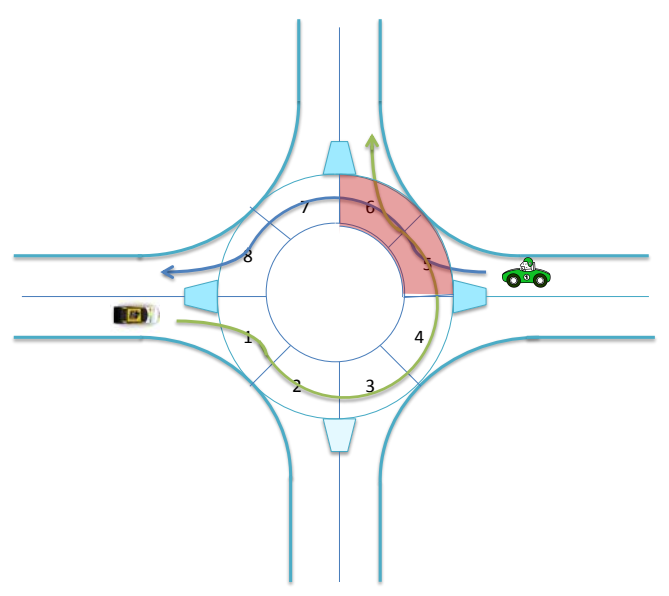

(b)

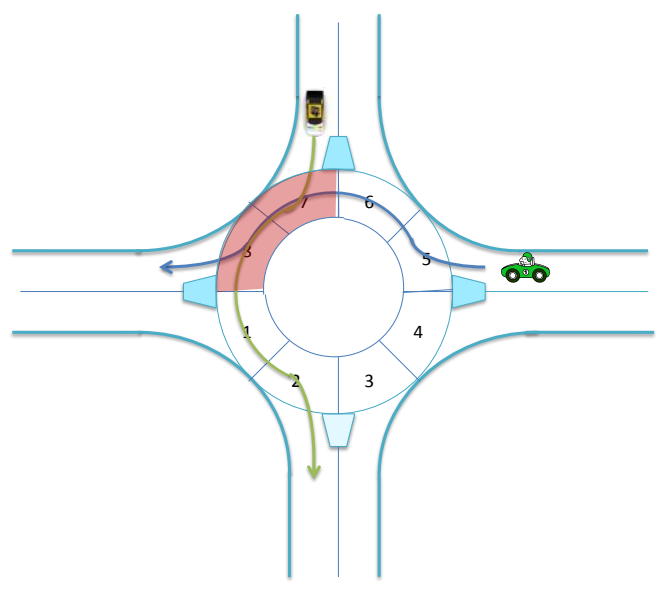

Page 4 of 13 
In the case that a potential collision is detected by CDAR, the "first come, first served" (FCFS) algorithm is used to assign priorities to vehicles. Based on FCFS, a vehicle, which gets to the entrance of the roundabout with a lower arrival time value, gets to cross the roundabout before other vehicles with higher arrival times. To avoid any deadlock situation, in which two or more vehicles have the same arrival time, tie-breaking rules apply. If vehicles arrive at a roundabout at the same time, our priority policy assigns higher priorities to vehicles entering the intersection using primary roads than vehicles arriving from secondary roads. If these still result in a tie among vehicles, the vehicle with a higher Vehicle ID Number (VIN) will have a higher priority and get to cross the roundabout grid first. The VIN is known unique for each vehicle.

\section{INTERSECTION PROTOCOLS}

In this section, we briefly describe our V2V-intersection protocols which were introduced in our previous work [12], and explain how these protocols have been adopted to be used for coordinating the traffic through roundabouts. These protocols include: (1) Concurrent Crossing-Intersection Protocol (CC-IP) and (2) Maximum Progression-Intersection Protocol (MP-IP). We have assumed that, in all our protocols, all vehicles have the same shape and physical dimensions. The communication medium has been assumed in this paper to have no packet loss 1 .

In our intersection protocols, each vehicle uses three types of intersection safety messages; ENTER, CROSS and EXIT, to interact with other vehicles in its communication range.

1. An ENTER message is used to inform neighboring vehicles that the vehicle is approaching the roundabout area with specific crossing intentions. The ENTER message contains 9 parameters: Vehicle ID, Current Road Segment, Current Lane, Next Road Segment, Next Vertex, Arrival-Time, Exit-Time, Trajectory Cells List, Message Sequence Number and Message Type, which is ENTER in this case.

2. A CROSS message is sent to inform that the vehicle is inside the roundabout grid. This message contains the sender's identification and trajectory details, identifying the space that will be occupied by the vehicle while crossing the roundabout. The CROSS message contains the same parameters as the ENTER message. Its Trajectory Cells List contains the updated list of trajectory cells, including the current cell and remaining cells along the vehicle's trajectory through the intersection area, and the Message Type: CROSS.

3. An EXIT message indicates that the vehicle has exited the roundabout boundaries. The EXIT message contains 3 parameters: Vehicle ID, Message Sequence Number, and Message Type: EXIT.

Every vehicle uses its own GPS coordinates, speed and also the map database to compute the distance to the approaching roundabout and the distance from the previous roundabout. We consider three states for each vehicle based on its relative location to the intersection area.

- INTERSECTION-APPROACH: when vehicle's distance to the approaching roundabout is less than a threshold parameter $D_{E N T E R}$.

- INTERSECTION-ENTER: when the vehicle is inside the roundabout grid's boundaries.

- INTERSECTION-EXIT: when the vehicle exits the roundabout, until it travels farther than a threshold value $D_{E X I T}$ from the exit point of the roundabout.

\subsection{Concurrent Crossing-Intersection Protocol (CC-IP)}

\footnotetext{
${ }^{1}$ In a study beyond the scope of this paper, we found that packet loss in NOT a problem in practical V2V scenarios.
} 
This protocol was designed to increase the throughput at intersections while avoiding collisions [12]. This intersection management protocol is based on pure $\mathrm{V} 2 \mathrm{~V}$ communications. The following send and receive rules are applicable to all vehicles:

\section{Algorithm 1: CC-IP Sender Vehicle}

Input: Vehicle's STATE based on its distance to the intersection

Output: Broadcasting the appropriate intersection safety message

1. If $\left(S T A T E=I N T E R S E C T I O N \_A P P R O A C H\right)$ then

2. Broadcast ENTER message

3. Else if ( STATE $=$ INTERSECTION_ENTER $)$ then

4. Broadcast CROSS message

5. Else if $($ STATE $=$ INTERSECTION_APPROACH $)$ then

6. Broadcast EXIT message

\section{Algorithm 2: CC-IP Receiver Vehicle}

Input: Received intersection safety message

Output: Vehicle's movement at the roundabout

1. If (received message $=$ ENTER), then

2. Run CDAR to detect trajectory conflicts with the sender

3. If (no potential collision is detected) then

4. Cross the roundabout

5. Else

6.

Use FCFS priority policy to determine the priority based on vehicles' arrival times

7.

If (higher priority than the sender) then

8.

Cross the roundabout

9. $\quad$ Else

10. Stop and wait to receive the EXIT message from the same sender

11. Else if (received message $=$ CROSS $)$ then

12. Run CDAR to detect trajectory conflicts with the sender

13. If (potential collision is detected) then

14. Stop and wait to receive the EXIT message from the same sender

15. Else

16. Check other receiving vehicles in the same condition*

17. If (higher priority or no conflict with higher priority vehicles) then

$18 . \quad$ Cross the roundabout

19. $\quad$ Else

20. Stop and wait to receive the EXIT message from the same sender

21. Else if (received message $=$ EXIT) then

22. If (the sender is the same sender of the last processed ENTER/CROSS message) then

23. Cross the roundabout

*: There might be more than one vehicle which has no conflict with the crossing vehicle at the same time. In this situation, these vehicles may attempt to cross the intersection area without being aware that they may collide with each other. Figures 7(a) and 7(b), show two situations in which vehicle $\mathrm{A}$ is crossing the roundabout and is broadcasting the CROSS message. Vehicles $\mathrm{B}$ and $\mathrm{C}$ are receiving these safety messages and run the CDAR algorithm. Both vehicles get to the same decision that they do not have a potential collision with vehicle A.

Page 6 of 13 
In Figure 7(a), vehicle B and C may still collide as they have a conflicting trajectory along the roundabout. So, only one of them can safely cross through the roundabout area while vehicle A is crossing, and the other vehicle will come to a complete stop before entering the roundabout boundaries. Vehicles $\mathrm{B}$ and $\mathrm{C}$ will reach to a decision using the priorities assigned to them by the priority policy so that the vehicle with the higher priority can cross, while the other waits outside the roundabout till it receives the EXIT message from the higher priority vehicle.

In Figure 7(b), vehicles B and C can cross the roundabout at the same time as vehicle A, since none of them has a conflict with the other two. So, the receiving vehicles make sure that they do not have trajectory conflicts with each other before entering the roundabout area. As discussed before, they may use the received ENTER messages and detect any potential collisions with other receivers of the CROSS message, which are waiting to enter the roundabout area. If no potential collision is detected with all other leader vehicles or if it has the highest priority among them, the receiver can cross the roundabout safely while broadcasting the CROSS message.

(a)

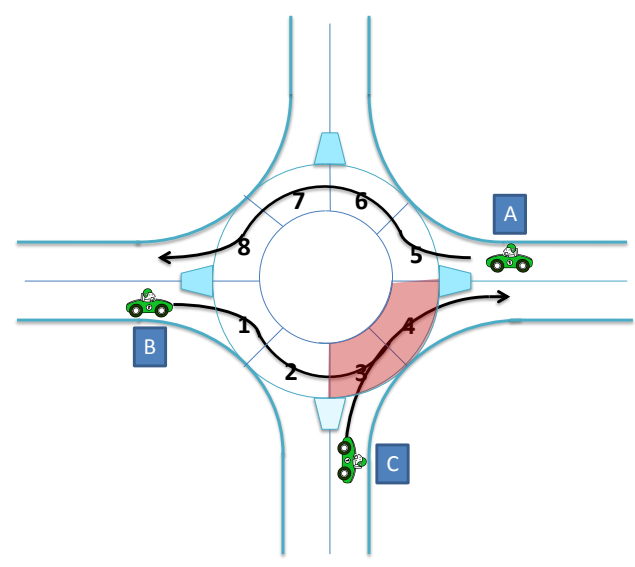

(b)

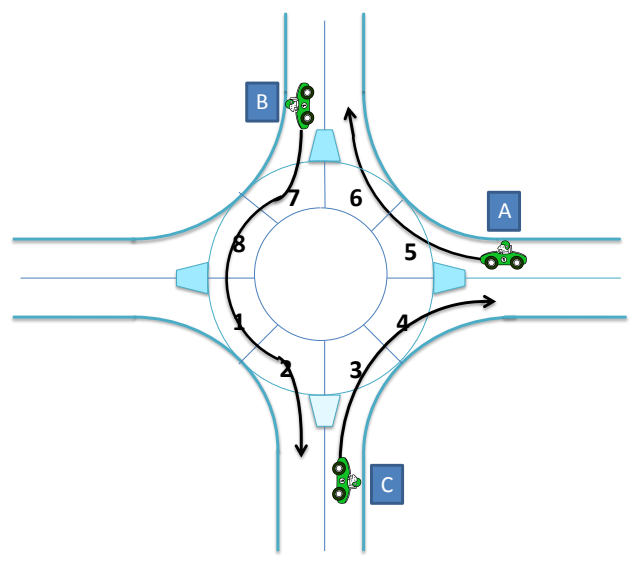

Figure 7, Two examples scenarios for CC-IP

\subsection{Maximum Progression-Intersection Protocol (MP-IP)}

As can be seen, $C C$-IP can be pretty conservative since no more than one vehicle with a conflicting trajectory can be inside an intersection at any given time. $M P-I P$ is a protocol that reduces the degree of conservatism significantly while satisfying all safety conditions. $M P-I P$ was designed to increase the throughput by allowing even conflicting vehicles to make maximal progress inside the intersection area, without sacrificing the primary goal of safety [12]. This V2V protocol makes use of the Trajectory Cells List (TCL) and our new Collision Detection Algorithm for Roundabouts (CDAR). The ENTER message contains a full list of cell numbers, TCL, which will be occupied by the vehicle along its trajectory crossing the intersection area. Same as in ENTER message, the CROSS message contains the $T C L$, but the list of cell numbers attached to this message changes as the vehicle makes progress inside the intersection box. At any moment of a vehicle's progress through the roundabout, the updated TCL contains the current cell number occupied by the vehicle and the remaining cells on its trajectory. The updated TCL is broadcast within the CROSS safety message.

The following rules are applicable to all vehicles:

\section{Algorithm 3: MP-IP Sender Vehicle}

Input: Vehicle's STATE, based on its distance to the intersection

Output: Broadcasting the appropriate intersection safety message

1. If $\left(S T A T E=I N T E R S E C T I O N_{-} A P P R O A C H\right)$ then

2. Broadcast ENTER message

3. Else if (STATE = INTERSECTION_ENTER $)$ then

4. Broadcast CROSS message

5. Else if (STATE $=$ INTERSECTION_APPROACH) then

6. Broadcast EXIT message

Page 7 of 13 


\section{Algorithm 4: MP-IP Receiver Vehicle}

Input: Received intersection safety message

Output: Vehicle's movement at the roundabout

1. If (received message $=$ ENTER or Received message $=$ Cross) then

2. Run CDAR to detect trajectory conflicts with the sender

3. If (no potential collision is detected) then

4. Cross the roundabout

5. Else

6.

Use FCFS priority policy to determine the priority based on vehicles' arrival times

7. If (higher priority than the sender) then

8.

Cross the roundabout

9.

Else

10. Find the TIC and progress up to it and get to a complete stop before entering the TIC and wait for receiving the updated message from the same sender to indicate the clearance of that TIC

11. Else if $($ Received message $=$ EXIT $)$ then

12. If (the sender is the same sender of the last processed ENTER/CROSS message) then

13. Cross the roundabout

In MP-IP, the vehicle which has the lower priority uses CDAR and finds out the first common cell (Trajectory Intersecting Cell) with the crossing higher-priority vehicle. Then, in contrast to CC-IP, instead of not entering the roundabout, it progresses inside the roundabout area and stops before entering the TIC. As soon as that cell becomes clear, the vehicle can proceed. This allows more vehicles to be inside the roundabout grid at the same time and decreases their trip delays.

Figure 8 shows two vehicles A and B approaching a roundabout. Assume that vehicle A has a higher priority than vehicle B. In this case, vehicle A gets to cross the roundabout without stopping or even slowing down. Vehicle B progresses inside the roundabout grid and stops before entering the first TIC with vehicle A, which is cell number 3. As vehicle A leaves cell number 3, it updates its TCL to $\{4\}$ and sends a CROSS message. This informs vehicle B that the TIC 3 is now clear and it can progress into that cell. Vehicle B's TCL gets updated to $\{3,4\}$ and cell number 4 is now the new common cell between A and B's TCLs. Since cell number 4 is occupied by vehicle $\mathrm{A}$, vehicle $\mathrm{B}$ cannot progress into cell number 4 until it receives an updated message from A indicating that cell 4 is no longer occupied by vehicle A.

(a)

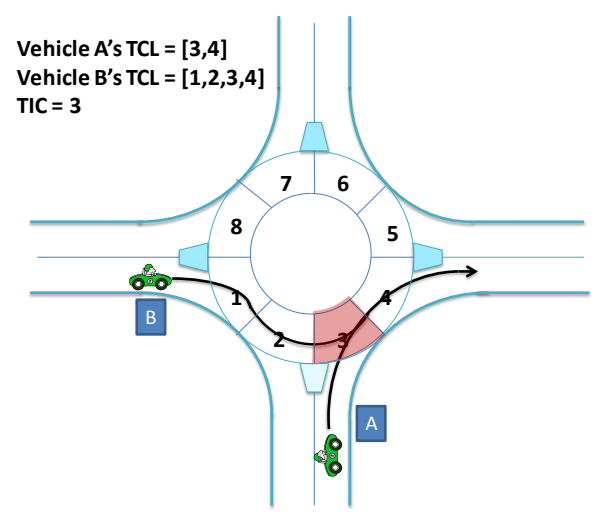

(b)

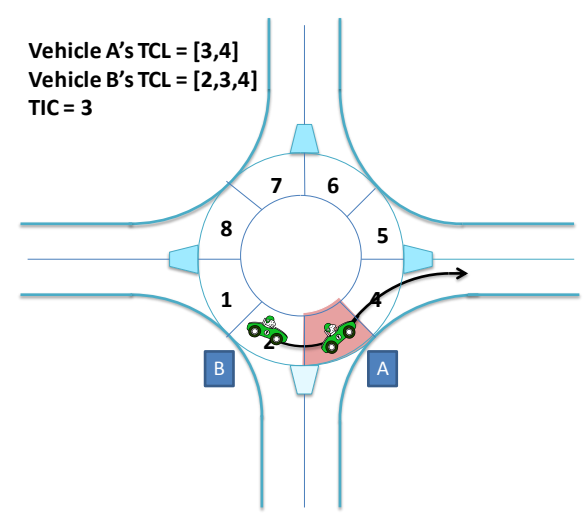


(c)

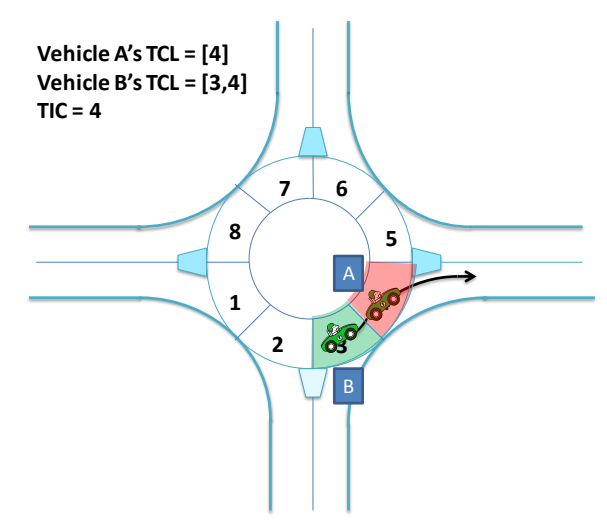

(d)

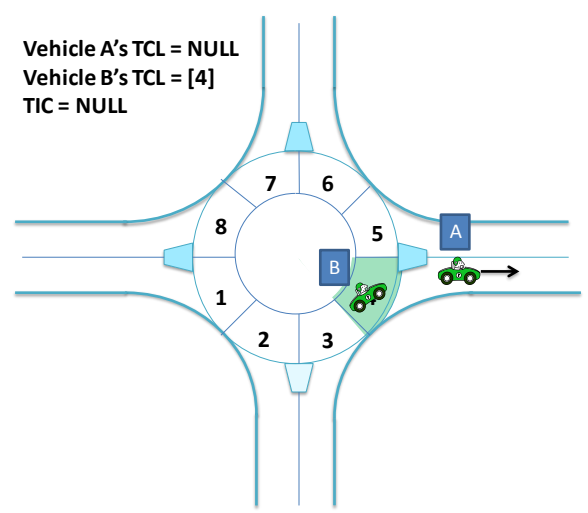

Figure 8, an example scenario for MP-IP

\section{PROTOCOL EVALUATION}

In this section, we evaluate our proposed protocols, CC-IP and MP-IP for roundabouts. We have used real GPS coordinates from map databases to generate Route Network Definition File (RNDF) files for roundabouts which already exist in the USA. Additionally, we have designed new mobility models to implement our V2V-intersection protocols within the roundabout area. All these models and roundabout RNDFs have been implemented in AutoSim. AutoSim is a hybrid emulation and simulation environment for vehicular communications. This emulator is a new next-generation version of GrooveNet $[9,10]$, and enables the interaction among real and simulated vehicles, and provides modeling of different aspects of mobility protocols. The architecture consists of several models such as the Control, Communication, Mobility, Localization and Path Tracking. Mobility models include the stop sign, traffic light, our V2V-based intersection models and the V2V car-following model. AutoSim also supports modeling of Vehicle-to-Vehicle (V2V) and Vehicle-to-Infrastructure (V2I) communications.

\subsection{METRIC}

We define the trip time for a vehicle as the time taken by that vehicle to go from a fixed starting point before the intersection to a fixed end point after the intersection. We calculate the trip time for each simulated car under each model and compare that against the trip time taken by the car assuming that it stays at a constant street speed and does not stop at the intersection. The difference between these two trip times is considered to be the Trip Delay due to the intersection. We take the average trip delays across all cars in a simulation sequence as our metric of comparison.

The trip route for each car is calculated using the Djikstra's Trip Model in AutoSim which calculates the shortest route between two points using Djikstra's algorithm [17]. The route is chosen with a waypoint at the intersection forcing the route to pass through the intersection. A logging mechanism has been added to AutoSim to enable logging of start and end times of cars to measure their trip times.

\subsection{SCENARIOS}

We run our simulations on 1-lane and 2-lane roundabouts, as well as 1-lane and 2-lane signalized perfect cross intersections. The roundabout/intersection type, vehicle-birthing sequence, vehicle routes and turn-types are generated offline. Each vehicle is removed from simulation when it reaches its destination. This file is then fed into AutoSim to invoke the intersection protocols. Each simulation run uses 1000 vehicles, and each run is terminated when the last vehicle reaches its destination.

\subsection{EXPERIMENTAL RESULTS}

In this experiment, we compare our $\mathrm{V} 2 \mathrm{~V}$ mobility models for a 1-lane roundabout, in which one-lane roads are entering the roundabout area from all four main directions. The amount of traffic volume is equal in each direction of the roundabout. We can see this comparison in Figure 9. As we can expect, Maximum Progression Intersection Protocol $(M P-I P)$ is more suitable for roundabouts and significantly outperforms Concurrent Crossing Intersection Protocol (CC-IP). The reason is that MP-IP allows vehicles to enter the roundabout as soon as there is an unoccupied cell and progress up to the conflicting cell. However, in the case of $C C$ - $I P$ vehicles will enter the roundabout only when they do not have any trajectory conflicts with the already crossing vehicle; otherwise they stay stopped outside of the roundabout and wait for the other vehicle to complete its passage inside the roundabout and exit it, which increases the delay.

Page 9 of 13 


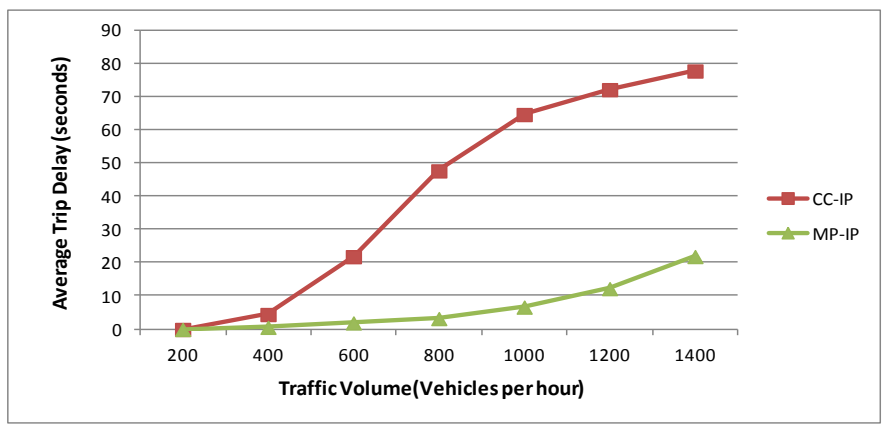

Figure 9, Delay comparison for 1-lane roundabouts managed by V2V models.

We then have replaced the same roundabouts with perfect cross intersections and have compared the trip delays in the case that the cross intersections are signalized. Two traffic light models have been used. One model has the green light duration of 10 seconds and the other one with the green light duration of 30 seconds per each direction. Figure 10 shows the results are based a wide range of traffic volumes; from 200 to 1400 vehicles per hour.

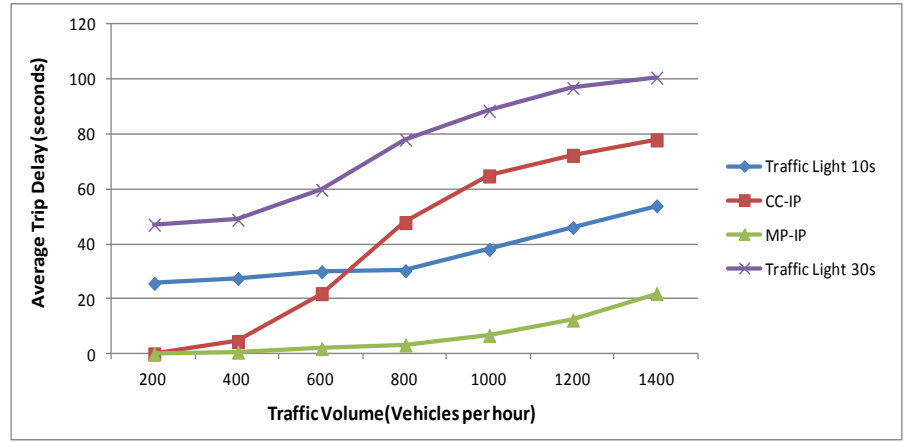

Figure 10, Delay Comparison between 1-lane intersections; V2V-roundabout models and signalized cross intersections.

According to the queueing theory, the average delay will asymptotically become very high when the arrival rate (i.e. traffic intensity) exceeds the service rate (throughput) at the intersection. This delay, however, occurs under steady-state conditions only after a considerable amount of time. Due to practical considerations, our simulations are run for finite durations, and hence capture only transient delay behaviors after overload conditions have been reached. Nevertheless, our results clearly indicate that before overload conditions are reached, the service rate (i.e. throughput) with the Maximum Progression Intersection Protocol (MP-IP) is noticeably better than the Traffic-Light models. MP-IP decreases the average trip delays by $\underline{80.91 \%}$ and $\underline{71.32 \%}$, respectively comparing to the traffic light models with 30 seconds and 10 seconds of green light durations.

We then repeated the above experiment for a 2-lanes roundabout and the corresponding results are shown in Figures 11 and 12 . Figure 11 shows that $M P-I P$ is still outperforming the $C C-I P$, but the gap has been reduced to $\underline{47.1 \%}$. The reason is that when dealing with a 2-lanes roundabout, $C C$-IP allows more vehicles to cross the roundabout concurrently and it does not get to the saturation point even when dealing with higher traffic volumes.

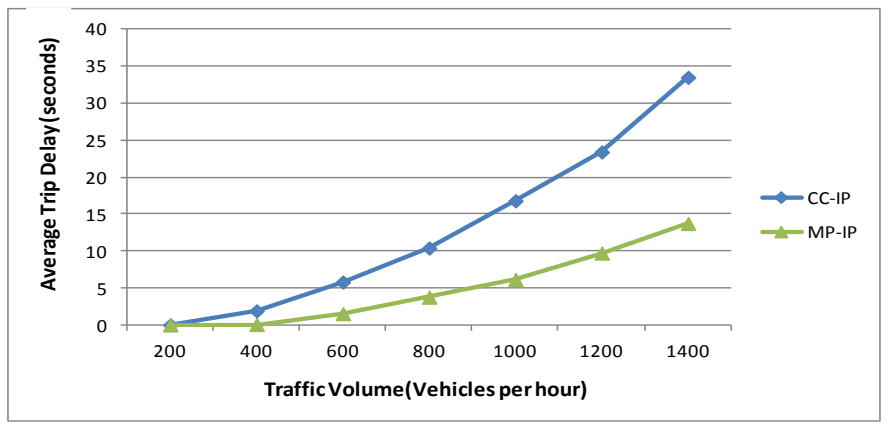

Figure 11, Delay comparison for 2-lanes roundabouts managed by V2V models. 
Figure 12 shows the scenarios that 2-lanes are entering the intersection from each direction. Our results indicate that V2V models outperform the traffic light models, as the number of conflicting points are significantly high in a 2-lanes cross intersection and this leads to higher delays specially for the vehicles which are attempting to make left turns. MP-IP model has negligible delay for low and medium traffic volumes and the average delay for very high traffic density is less than 15 seconds. MP-IP decreases the average trip delays by $\underline{90.29 \%}$ and $\mathbf{7 5 . 8 8 \%}$, respectively comparing to the traffic light models with 30 seconds and 10 seconds of green light durations.

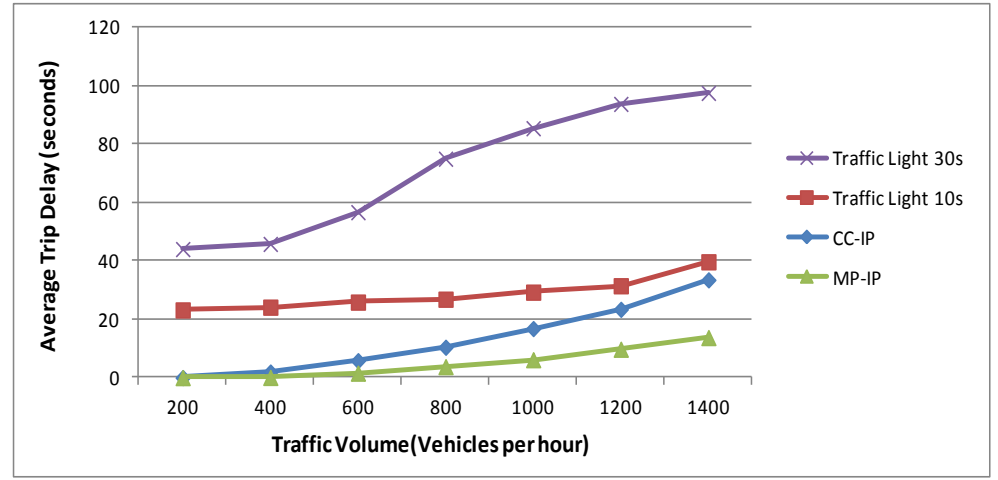

Figure 12, Delay Comparison between 2-lanes intersections; V2V-roundabout models and signalized cross intersections.

We finally have simulated the scenarios in which the traffic is asymmetric and the traffic volume on north and south legs are significantly higher than east and west legs of the intersection/roundabout. Figure 13 shows comparisons of average trip delays, for 2lane signalized cross intersections and 2-lane roundabouts in cases of symmetric and asymmetric traffic flows. Note that the passage of vehicles through the roundabout is coordinated with MP-IP. When dealing with asymmetric traffic in a signalized intersection, vehicles which are approaching the intersection from high density roads face much longer delays and this increases the overall average delay of the intersection and decreases the throughput. We can see that for asymmetric traffic the average delays increase exponentially as the traffic volume increases and gets closer to the saturation phase However, it can be seen that in an MP-IP-managed roundabout, even though the delays are increased in asymmetric traffic, but the increase is very negligible and almost zero for low and medium traffic volumes.

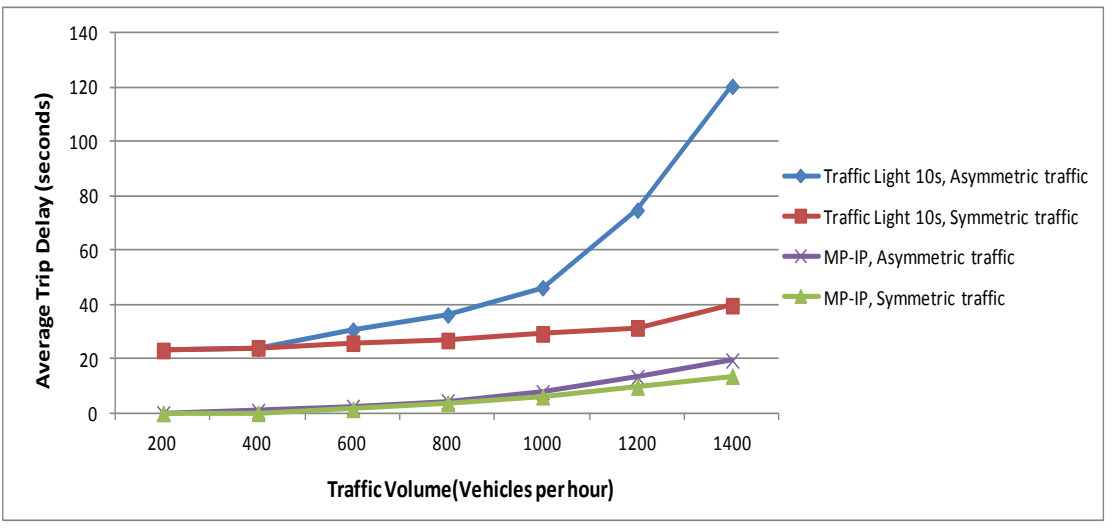

Figure 13, Delay Comparison for symmetric and asymmetric traffic. MP-IP-roundabout models and signalized cross intersections.

\section{CONCLUSIONS}

A substantial fraction of automotive collisions occur at intersections. Current infrastructures such as stop signs and traffic lights are believed to be the bottlenecks of the traffic which increase the trip times significantly. In this paper, our goal was to improve our V2Vbased intersection management protocols and implement them on roundabouts. We also introduced a new Collision Detection Algorithm for Roundabouts (CDAR) to avoid collisions at roundabouts. We extended our emulator-simulator AutoSim, by introducing Route Network Definition File (RNDF) files and constructing roundabout routes based on the GPS coordinates extracted from digital map databases. While statistics collected by Insurance Institute for Highway Safety (IIHS) show that when comparing roundabouts to signalized intersections, delays are reduced up to $20 \%$, our results indicate that by using V2V communications as a

Page 11 of 13 
part of co-operative driving, we are able to reduce the delays even more. By using our proposed V2V-Intersection protocol; Maximum Progression Intersection Protocol (MP-IP) to coordinate the traffic through roundabouts, we are able to reduce the delays up to $75.88 \%$ comparing to various signalized intersections. [15]. MP-IP avoids collisions inside the roundabout area, as it prevents vehicles to enter the conflicting cells which are reserved by higher priority vehicles and increases the traffic throughput by allowing more vehicles to benefit from the gaps in the traffic flow, enter the roundabout at the same time and cross concurrently. Although our protocols are designed for autonomous vehicles that use V2V communication for co-operative driving, they can be adapted to a driveralert system for manual vehicles at traffic intersections.

We intend to overcome the limitations described above and extend the V2V protocols in the context of real cars. We are working on hybrid simulations with real and simulated vehicles to take advantage of AutoSim's hybrid environment. We have indeed already implemented a version of this protocol on real-world Segway robots but it is not captured in this paper. There is also ongoing work to look at integration of Vehicle-to-Infrastructure (V2I) technologies within these protocols to take advantage of statically known entities at intersections. We intend to design new protocols which use the integration of V2I and V2V for managing intersections, where autonomous and human-driven vehicles are both present.

\section{References}

1. US Department of Transportation-Federal Highway Administration Publication, National Agenda for Intersection Safety http://safety.fhwa.dot.gov/intersection/resources/intersafagenda/

2. Seyed Reza Azimi, Gaurav Bhatia, Ragunathan (Raj) Rajkumar, Priyantha Mudalige Vehicular Networks for Collision Avoidance at Intersections, Society for Automotive Engineers (SAE) World Congress,April,2011

3. Kurt Dresner \& Peter Stone (2008), Replacing the Stop Sign: Unmanaged Intersection Control, the Fifth Workshop on Agents in Traffic and Transportation Multiagent Systems. pp. 94-101, Estoril, Portugal.

4. Kurt Dresner \& Peter Stone (2008), A Multiagent Approach to Autonomous Intersection Management. Journal of Artificial Intelligence Research(JAIR)

5. V. Drabkin, R. Friedman, G. Kliot, and M. Segal. Rapid: Reliable probabilistic dissemination in wireless ad-hoc networks. In The 26th IEEE International Symposium on Reliable Distributed Systems, Beijing, China, October 2007.

6. Vehicle Safety Communications 2 consortium. Vehicle Safety Communications - Applications (VSC-A) Final Report. Washington, DC: National Highway Traffic Safety Administration

7. Laurent Bouraoui, Stéphane Petti, Anis Laouiti, Thierry Fraichard and Michel Parent, INRIA Rocquencourt, "Cybercar cooperation for safe intersections", Proceedings of the IEEE ITSC, 2006 IEEE Intelligent Transportation Systems Conference, Toronto, Canada, September 17-20, 2006

8. 2010 0-60mph statistics. [Online]. Available at: http://www.0-60cartimes.com/0-60-statistics

9. R. Mangharam, J. Meyers, R. Rajkumar, D. Stancil, J. Parikh, H. Krishnan, and C. Kellum, "A Multi-hop Mobile Networking Test-bed for Telematics" Society for Automotive Engineers (SAE) World Congress. Detroit, USA. April 2005

10. R. Mangharam, D. S. Weller, R. Rajkumar, P. Mudalige and Fan Bai, "GrooveNet: A Hybrid Simulator for Vehicle-toVehicle Networks", Second International Workshop on Vehicle-to-Vehicle Communications (V2VCOM), San Jose, USA. July 2006

11. Draft SAE J2735_200911 Dedicated Short Range (DSRC) message set dictionary.

12. Seyed Reza Azimi, Gaurav Bhatia, Ragunathan (Raj) Rajkumar, Priyantha Mudalige, "Intersection Management Using Vehicular Networks", Society for Automotive Engineers (SAE) World Congress,April,2012

13. Michigan Department of Transportation. http://www.michigan.gov/roundabouts

14. Washington State, Department of Transportation. http://www.wsdot.wa.gov/Safety/roundabouts/benefits.htm

15. IIHS, Insurance Institute for Highway Safety, Status Report, Vol. 36, No. 7, July 28, 2001.

16. Hummer, J.E., Chapter 14: Intersection and Interchange Design, in Handbook of Transportation Engineer. p. pp. 14.1$14.27,2004$.

17. Dijkstra, E. W. (1959). "A note on two problems in connexion with graphs". Numerische Mathematik 1: $269-271$.

18. US Department of Transportation- National Highway Traffic Safety Administration. Traffic Safety Facts 2009. http://www-nrd.nhtsa.dot.gov/Pubs/811402.pdf

19. Vehicle Safety Communications 2 consortium. Cooperative Intersection Collision Avoidance System Limited to Stop Sign and Trafic Signal Violations (CICAS-V) Final Report. Washington, DC: National Highway Traffic Safety Administration.

20. Ahmed-Zaid, F., Bai, F., Bai, S., Basnayake, C., Bellur, B., Brovold, S., Brown, G., Caminiti, L., Cunningham, D., Elzein, H., Ivan, J., Jiang, D., Kenny, J., Krishnan, H., Lovell, J., Maile, M., Masselink, D., McGlohon, E., Mudalige, P., Rai, V., Stinnett, J., Tellis, L., Tirey, K., VanSickle, S. Vehicle Safety Communications - Applications (VSC-A) First Annual Report. Washington, DC: National Highway Traffic Safety Administration.

Page 12 of 13 
21. Shulman, Mike; Deering, Richard, K., "Crash Avoidance Metrics Partnership (CAMP) - Second Annual Report", Sponsored by NHTSA, Washington D.C., April 2002 - March 2003, DOT HS 809663.

22. Nevada Department of Transportation, Federal Highway Administration Publication. http://www.nevadadot.com/safety/roundabout/benefits.aspx

\section{CONTACT INFORMATION}

Reza Azimi: rezaazimi@.cmu.edu

Gaurav Bhatia: gnb@ece.cmu.edu

Ragunathan (Raj) Rajkumar: raj@ece.cmu.edu

Priyantha (Pri) Mudalige: priyantha.mudalige@gm.com 